\title{
Combined Immunodeficiency Caused by a Novel De Novo Gain-of-Function RAC2 Mutation
}

\section{Xiaodong Zhao ( $\sim$ zhaoxd530@aliyun.com )}

Children's Hospital of Chongqing Medical University https://orcid.org/0000-0003-2054-8711

\section{Liang Zhang}

Children's Hospital of Chongqing Medical University

\section{Zhi Chen}

Children's Hospital of Chongqing Medical University

\section{Wenyan Li}

Children's Hospital of Chongqing Medical University

\section{Qiao Liu}

Children's Hospital of Chongqing Medical University

\section{Yanping Wang}

Children's Hospital of Chongqing Medical University

\section{Xuemei Chen}

Children's Hospital of Chongqing Medical University

\section{Zhirui Tian}

Children's Hospital of Chongqing Medical University

\section{Qiuyun Yang}

Children's Hospital of Chongqing Medical University

\section{Yunfei An}

Children's Hospital of Chongqing Medical University

\section{Zhiyong Zhang}

Children's Hospital of Chongqing Medical University

\section{Huawei Mao}

Children's Hospital of Chongqing Medical University

\section{Xuemei Tang}

Children's Hospital of Chongqing Medical University

\section{Ge LV}

Children's Hospital of Chongqing Medical University https://orcid.org/0000-0003-3763-1933

\section{Research Article}

Keywords: Ras-related C3 botulinum toxin substrate 2 (RAC2), combined immunodeficiency, apoptosis, polarization

Posted Date: February 11th, 2022

DOI: https://doi.org/10.21203/rs.3.rs-1303580/v1

License: (c) (i) This work is licensed under a Creative Commons Attribution 4.0 International License. Read Full License 


\section{Abstract}

Ras-related C3 botulinum toxin substrate 2 (RAC2) is a GTPase exclusively expressed in hematopoietic cells, that acts as a pivotal regulator of several aspects of cell behavior via various cellular processes. RAC2 undergoes a tightly-regulated GTPbinding/GTP-hydrolysis cycle, enabling it to function as a molecular switch. Mutations in RAC2 have been identified in 18 patients with different forms of primary immunodeficiency, ranging from phagocyte defects caused by dominant negative mutations to common variable immunodeficiency resulting from autosomal recessive loss-of-function mutations, or severe combined immunodeficiency due to dominant activating gain-of-function mutations. Here, we describe an 11-year-old girl with combined immunodeficiency presenting with recurrent respiratory infections and bronchiectasis. Immunological investigations revealed low T-cell receptor extension circle/K-deleting recombination excision circles numbers, lymphopenia, and low serum immunoglobulin G. Targeted next generation sequencing identified a novel heterozygous mutation in RAC2, c.86C $>$ G (p.P29R), located in the highly-conserved Switch I domain. The mutation resulted in enhanced reactive oxygen species production, elevated F-actin content, and increased RAC2 protein expression in neutrophils, as well as increased cytokine production and a dysregulated phenotype in T lymphocytes. Further, the dominant activating RAC2 mutation led to accelerated apoptosis with augmented intracellular active caspase 3 , impaired actin polarization in lymphocytes and neutrophils, and diminished RAC2 polarization in neutrophils. We present a novel RAC2 gain-of-function mutation with implications for immunodeficiency and linked to functional dysregulation, including abnormal apoptosis and cell polarization arising from altered RAC2 expression. Thus, our findings broaden the spectrum of known RAC2 mutations and their underlying mechanisms.

\section{Introduction}

RAC2 is a hematopoietic cell-specific member of the Ras homology (Rho) family of guanosine triphosphatases (GTPases) that functions as a crucial regulator of cell signaling and actin dynamics. RAC2 has roles in numerous important functions, including roles in gene transcription, cell survival, cell adhesion, reactive oxygen species (ROS) production, and cytoskeleton reorganization [1]. Progress in exploring RAC2 function and regulation in mice has facilitated understanding of its physiological roles; for example, regulation of hematopoietic stem cell interaction within the bone marrow niche, erythropoiesis, red blood cell actin dynamics, and phagocyte migration and killing, as well as T and B cell maturation [2]; however, RAC1, which is highly homologous with RAC2, is ubiquitously expressed, and the two proteins are co-expressed in mouse cells of hematopoietic origin. In this context, RAC1 and RAC2 are likely to show functional redundancy, which may obscure their specific roles. Nevertheless, in vivo studies have demonstrated that RAC2 coordinates unique cellular processes [3-7].

In recent years, different RAC2 mutations have been identified and reviewed in a small number of patients, revealing further information regarding the vital, non-redundant, and complex functions of RAC2 in humans [8-11]. A dominant negative monoallelic mutation, p.D57N, classified as causing congenital phagocyte defects, was first described in a male infant in 2000. The infant presented with major symptoms including recurrent progressive soft-tissue infections and wound healing failure, mainly resulting from dysregulated neutrophil function, including defective migration, polarization, and reduced superoxide generation [12-14]. Another patient with the p.D57N mutation, identified by screening of newborns for severe combined immunodeficiency, initially presented with T-cell lymphopenia, further indicating that RAC2 has complex functional roles $[15,16]$. Subsequently, two siblings were found to be homozygous for the recessive null RAC2 allele, p.W56X, leading to common variable immunodeficiency, along with T-cell compartment abnormalities. These patient phenotypes were qualitatively distinct from the defects observed in $\mathrm{RAC2}^{-/-}$mice to some extent, indicating specific roles for RAC2 in human hematopoietic cells [17].

The nucleotide-bound state of the RAC2 GTPase is tightly regulated as a molecular switch that undergoes a GTPbinding/GTP-hydrolysis cycle dominated by guanine nucleotide exchange factors (GEFs) and GTPase activating proteins (GAPs) [18]. Since 2019, several groups have reported germline monoallelic activating gain-of-function mutations in RAC2, 
including p.G12R, p.P34H, p.Q61R, p.E62K, and p.N92T. These hyperactivating mutations resulted in combined immunodeficiency (CID) or severe combined immunodeficiency (SCID) phenotypes, associated with severe lymphopenia, recurrent sinopulmonary infections, and invasive viral infections, or severe bone marrow hypoplasia and absence of circulating leukocytes [19-24], providing crucial insights into the roles of RAC2 in human leukocyte biology and helping to elucidate the significance of RAC2 modification.

To date, 18 patients with diverse RAC2 mutations leading to heterogeneous clinical phenotypes and immunological defects have been reported in the literature. The mechanisms underlying the effects of mutations in this gene, which can affect the immune system in various ways, remain unclear and require elucidation. Herein, we describe the clinical and immunological features of a Chinese patient with a previously unreported heterozygous mutation in RAC2, c.86C>G (p.P29R), including combined immunodeficiency, increased apoptosis, and decreased polarization of actin in lymphocytes and neutrophils.

\section{Materials And Methods}

\section{Patient}

The patient enrolled in this study was an 11-year-old girl born to a nonconsanguineous family. Clinical data and blood samples were collected when she first presented to the Children's Hospital of Chongqing Medical University in October 2017. This study was approved by the Medical Ethics Committee of the Children's Hospital of Chongqing Medical University (Approval number: 030/2013). Informed consent was obtained from the patient and her guardians.

\section{Genetic Analysis}

Whole blood samples were subjected to targeted gene sequencing of 269 primary immunodeficiency genes performed on Illumina HiSeq2000 platform (Illumina, San Diego, Calif) by MyGenostics (Beijing, China). Germline mutations in RAC2 were verified by Sanger sequencing, using the primer sequences: forward, 5囚-TAGGCTGGGTGGATGCTGAG-3\ and reverse, 5》TCCTCCATACCCCATCCCGG-3囚. RAC2 mRNA was amplified by real-time fluorescent quantitative PCR, following reverse transcription to CDNA, using SYBR Green core reagents on a CFX96Touch ${ }^{\text {TM }}$ system (Bio-Rad), according to the manufacturer's instructions, with the primer sequences: forward, 5囚-TCATCTGCTTCTCCCTCGT-3\ and reverse, 5囚-

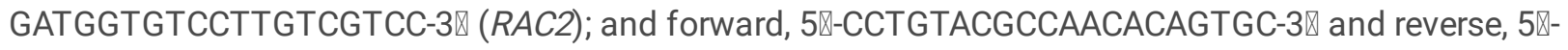
ATACTCCTGCTTGCTGATCC-3凶 (ACTB, encoding $\beta$ actin).

\section{Flow Cytometric Analysis of T and B Cells}

Circulating follicular helper T (cTfh) cells, Th1 cells, Th2 cells, Th17 cells, and subsets of cTfh cells were quantified in $50 \mu \mathrm{L}$ whole blood samples in separate experiments, as described previously [25]. B cell subpopulations were defined as documented elsewhere [26]. Samples were acquired on a FACSCanto II flow cytometer and data were analyzed using FlowJo software.

\section{Flow Cytometric Analysis of Neutrophils}

Peripheral blood neutrophils were isolated as recorded previously [27]. After isolation, granulocytes were kept on ice in KRG buffer ( $\mathrm{pH}$ 7.3; $120 \mathrm{mM} \mathrm{NaCl}, 5 \mathrm{mM} \mathrm{KCl}, 1.7 \mathrm{mM} \mathrm{KH}_{2} \mathrm{PO}_{4}, 8.3 \mathrm{mM} \mathrm{NaH}_{2} \mathrm{PO}_{4}, 10 \mathrm{mM}$ glucose), supplemented with $\mathrm{Ca}^{2+}$ (1 $\mathrm{mM})$ and $\mathrm{Mg}^{2+}(1.5 \mathrm{mM})$. For assessment of neutrophil activation and degranulation, cells were left untreated at room temperature or incubated for $20 \mathrm{~min}$ at $37^{\circ} \mathrm{C}$ with or without N-Formylmethionyl-leucyl-phenylalanine (fMLP) (50 nM; Sigma-Aldrich), tumor necrosis factor a (10 ng/mL; Sigma-Aldrich), or WKYMVM peptide (50 nM; Sigma-Aldrich). Granulocytes were fixed and erythrocytes lysed by treatment with FACS lysing solution (BD Biosciences) for 15 min at $4^{\circ} \mathrm{C}$. 
Cells were then stained with anti-CD11b, anti-CD62L, anti-CD35, anti-CD63, or anti-CD66b antibodies (all from BD Biosciences) for $1 \mathrm{~h}$ on ice. A minimum of 10,000 neutrophils were assessed by flow cytometry [28].

\section{T-Cell Proliferation Analysis}

Peripheral blood mononuclear cells were incubated with $1 \mathrm{mM}$ carboxyfluorescein succinimidyl ester (CFSE) (Invitrogen/ThermoFisher Scientific), as previously described [25], and seeded in 96-well plates with $5 \mathrm{mg} / \mathrm{mL}$ phytohemagglutinin (Sigma-Aldrich) or soluble aCD3/aCD28-coated beads (ThermoFisher Scientific) for $72 \mathrm{~h}$. After staining with anti-CD3, anti-CD4, and anti-CD8 antibodies (all from Biolegend), cells were analyzed using a FACSCanto II flow cytometer.

\section{Quantification of T-Cell Receptor Excision Circles}

Real-time quantitative PCR (RT-qPCR) to detect T-cell receptor excision circles (TRECs) and K-deleting recombination excision circles (KRECs) was performed as described previously [29]. Results are reported as numbers of TRECs and KRECs per $10^{6}$ cells or per $\mathrm{mL}$ of blood.

\section{Assay of Granulocyte Intracellular NADPH Oxidase Activity}

NADPH oxidase activity was measured by luminol/isoluminol-enhanced chemiluminescence (CL) using a microplate reader (Synergy H1 MultiMode Reader, BioTek, U.S.A) [30]. For intracellular ROS measurement, granulocytes were added to KRG$\mathrm{Ca}^{2+}$ containing cell permeable luminol $(10 \mu \mathrm{g} / \mathrm{mL}$; Sigma-Aldrich) and $50 \mathrm{U} / \mathrm{mL}$ superoxide dismutase (Solarbio) to scavenge extracellular superoxide, while extracellular ROS was measured in the presence of $10 \mu \mathrm{g} / \mathrm{mL}$ isoluminol (SigmaAldrich). Neutrophils and reagents were then incubated for $5 \mathrm{~min}$ at $37^{\circ} \mathrm{C}$ before stimulation with fMLP $(1 \mu \mathrm{M}$; SigmaAldrich), WKYMVM peptide (1 $\mu \mathrm{M}$; Sigma-Aldrich), or phorbol 12-myristate 13-acetate (PMA) (500 nM; Sigma-Aldrich). CL was recorded continuously for 20-25 min.

\section{Analysis of Actin Polymerization}

For immunofluorescence staining, CD3 T cells were isolated using an EasySep Human CD3 T Cell positive selection Kit (Stemcell), according to the manufacturer's instructions, then plated on poly-L-lysine-coated slides in the presence of soluble aCD3/aCD28-coated beads or $5 \mu \mathrm{g} / \mathrm{mL}$ low endotoxin azide free (LEAF)-purified anti-CD3 and anti-CD28 antibodies (Biolegend) for $30 \mathrm{~min}$ [31]. Isolated neutrophils were plated on slides in the presence of $500 \mathrm{nM}$ PMA (SigmaAldrich) [32]. After fixation and blocking, cells were stained with phalloidin (Molecular Probes) at a 1:40 dilution and 49-6diamidino-2-phenylindole dihydrochloride at 1:10,000 in PBS. Cover slips were imaged on a laser scanning confocal microscope (Nikon, Japan). For analysis of phalloidin mean fluorescence intensity [33], neutrophils were equilibrated for 5 min at $37^{\circ} \mathrm{C}$, followed by agonist stimulation, and cell suspensions transferred to ice-cold BD fixation/permeabilization solution at different time points post-stimulation and incubated on ice for 20 min. F-actin was stained with FITC-phalloidin for $30 \mathrm{~min}$ at $4^{\circ} \mathrm{C}$ in darkness. FITC-phalloidin intensity was analyzed using a FACSCanto II flow cytometer.

\section{Analysis of Apoptosis}

Apoptosis of peripheral T cells from controls or the patient was assessed with or without $5 \mu \mathrm{g} / \mathrm{mL}$ LEAF-purified anti-CD3 and anti-CD28 antibodies (Biolegend) or $10 \mu \mathrm{g} / \mathrm{mL}$ LEAF-purified FASL (Biolegend) for $48 \mathrm{~h}$ [34]. Apoptosis of B cells from controls or the patient was assessed following stimulation with $0.6 \mu \mathrm{g} / \mathrm{ml} \mathrm{CpG-ODN}$ (InvivoGen) and $5 \mu \mathrm{g} / \mathrm{mL}$ F(ab)2 goat 
anti-human IgM (Jackson ImmunoResearch) for $48 \mathrm{~h}$ [35]. Apoptosis of isolated neutrophils was examined with or without $0.1 \mu \mathrm{g} / \mathrm{mL}$ LPS (Sigma-Aldrich) or $10 \mu \mathrm{g} / \mathrm{mL}$ LEAF-purified FASL (Biolegend) for $16 \mathrm{~h}$ [32]. Cells were stained with Annexin V (to detect apoptosis) and 7-aminoactinomycin D (to detect necrosis). Live cells were identified by the absence of staining for either Annexin V or 7-AAD antibodies (both from BD Biosciences). Apoptosis was also evaluated by detection of intracellular expression of active caspase 3 by flow cytometric analysis [34].

\section{Immunostaining Analysis of RAC2}

Isolated neutrophils were plated on slides in the presence of $500 \mathrm{nM} \mathrm{PMA} \mathrm{(Sigma-Aldrich)} \mathrm{for} 30$ min [32], fixed with 4\% paraformaldehyde, permeabilized with $0.3 \%$ Triton X-100, and stained for RAC2 (clone: bs-6153R, 1:50 dilution; Bioss). Cells were counterstained with 49-6-diamidino-2-phenylindole dihydrochloride (1:10,000 in PBS). Cover slips were imaged on a laser scanning confocal microscope.

\section{Immunoblotting Analysis of RAC2}

Isolated neutrophils were lysed and subjected to sodium dodecyl sulfate/polyacrylamide gel electrophoresis on $12 \%$ gels and then transferred to PVDF membranes (Millipore). Blots were then rinsed and blocked in PBS-T containing 5\% non-fat dried milk (pH 7.5). After overnight incubation $\left(4^{\circ} \mathrm{C}\right)$ with anti-Rac2 (ab154711, Abcam) and anti- $\beta$-actin (SigmaAldrich), blots were incubated with horseradish peroxidase-conjugated anti-rabbit lgG (Cell Signaling Technology) and detected using Clarity Western ECL substrate (Bio-Rad).

\section{Statistical Analysis}

Data were analyzed using an unpaired two-tailed Student $t$ test. All statistical analyses were conducted using GraphPad Prism 8 software (GraphPad Software, Inc., San Diego, CA). P $\leq 0.05$ was considered to indicate a significant difference.

\section{Results}

\section{Patient Clinical Characteristics}

The patient was from a nonconsanguineous kindred with no significant family history. She first presented with fever and cough at 4 months and was diagnosed with pneumonia, and since had recurrent pneumonia at a frequency of three times each year, which was treated locally with repeated oral and intravenous antibiotics. She also experienced recurrent episodes of hemoptysis from 8 years old, with documented bronchiectasis. Due to the recurrent fever and pneumonia, requiring increasingly longer hospitalization and higher grade antibiotic treatment, our hospital was consulted when she was 10 years old; she weighed $20 \mathrm{~kg}$ and her height was $122 \mathrm{~cm}$ on admission. On clinical examination, clubbing of her fingers was detected, along with moderate moist rales and hepatosplenomegaly. Laboratory investigations showed constitutive severe lymphopenia and fluctuating granulocytopenia, which was particularly prominent during infection, with large vacuoles visible by light microscopy (Fig. 1a-d), and decreased serum immunoglobulin G (IgG) (Table 1), with no commonly positive autoantibodies, such as anti-nuclear, anti-neutrophil cytoplasmic, or anti-thyroid peroxidase antibodies. Haemophilus influenzae was repeatedly isolated from sputum on etiological examination, while fungal, cytomegalovirus (CMV), and Epstein-Barr virus (EBV) infections were absent. Chest computer tomography scan revealed extensive bronchiectasis (Fig. 1e). From the beginning of 2019, she began to receive regular intravenous immunoglobulin treatment each month; however, pulmonary infections continued to occur more than five times per year and required hospitalization for combined antibiotic treatment. 


\section{Immunophenotype}

The patient had severe T, B, and NK cell lymphopenia, with high relative percentages of effector/memory T cells, while a markedly reduced CD4:CD8 ratio, as well as recent thymic and bone marrow emigrants (Table 2). She also showed diminished proliferative responses to stimulation with mitogens or T-cell receptor (TCR), while cytokine production was elevated (Fig. S1 and Fig. S2). Further work-up revealed extended active cTfh, as well as increased percentages of Th1 and Th1/17-like subsets among non-cTfh and cTfh cells (Fig. 2a, b). Additionally, the patient had a significatively increased percentage of double-negative B cells, along with $\mathrm{CD} 21^{\text {low }} \mathrm{B}$ cells (Fig. 2c). Collectively, these results indicated combined immunodeficiency with a dysregulated phenotype.

\section{A Novel Heterozygous RAC2 Mutation Identified by Gene Sequencing}

Sequencing analysis using an NGS-based immunodeficiency panel revealed a novel de novo heterozygous variant in RAC2 (c.86C>G), which was confirmed by Sanger sequencing, and causes a proline to arginine substitution at position 29 (p.P29R) of the RAC2 protein (Fig. 3a, b). Pathogenicity analysis of this novel mutation predicted that it is deleterious (Table 3). The mutated P29 residue resides within a highly-conserved Switch I domain (Fig. 3c), which is vitally important for interactions with GEFs and downstream effectors [36]; however, 3D-modeling structural analysis of the mutant protein using Swiss PdbViewer did not show any obvious destruction of hydrogen bonds or influence on interactions with GDP (data not shown). Nevertheless, data on RAC2 and RAC1 mutations in cancer cells support the hypothesis that this novel mutation is disease causing; RAC1 p.P29S substitutions have been identified in sun-exposed melanomas, head and neck squamous cell carcinomas, as well as analogous RAC2 p.P29L and p.P29Q mutations [37-39], supporting the importance of this proline residue for normal Switch I region function. This variant meets the American College of Medical Genetics (ACMG) criteria for pathogenesis [40], as follows: pathogenic strong (PS1) evidence, as it is a de novo mutation; pathogenic moderate 1 (PM1), due to its location in a well-established functional domain; pathogenic moderate 2 (PM2), as the mutation is not reported in population databases, such as the 1000 Genomes Project; and pathogenic supporting 3 (PP3), since multiple lines of computational evidence support a deleterious effect. Taken together, these findings indicate that the variant is plausibly a novel pathogenic heterozygous $R A C 2$ mutation.

\section{Enhanced Neutrophil ROS Production and Activation}

RAC2 has non-redundant and crucial roles in multiple biological functions of neutrophils, including in oxidase activity [41]. Neutrophils from RAC2 ${ }^{-1-}$ mice and patients with the RAC2 p.D57N mutation exhibit impaired ROS production in response to fMLP, whereas patients with activating gain-of-function mutations, such as RAC2 p.E62K and p.P34H, show enhanced ROS generation $[19,20]$. Samples from our patient also showed increased ROS production in response to fMLP and WKYMVM, while levels were normal following PMA stimulation (Fig. 4a), since the latter is independent of RAC2 [19]. These findings were reminiscent of the enhanced ROS production and stimulation-induced apoptosis in BTK-deficient human neutrophils, resulting from increased RAC2 activity in the plasma membrane in a primed state, which has been proposed as responsible for neutropenia and tissue damage [32]. Further, neutrophil activation was quantified by multicolor flow cytometry to detect cell-surface CD11b and CD62L expression, and degranulation was assessed by analysis of cell surface expression of CD35 (secretory vesicle), CD63 (azurophilic granules), and CD66b (gelatinase granules). Samples from our patient showed increased neutrophil activation, but no obvious degranulation abnormalities in response to different stimuli (Fig. S3).

\section{Impaired Actin Polarization in T Cells and Neutrophils}

The activated state of the RAC2 GTPase is tightly regulated, and appropriate inactivation of RAC2 is required for F-actin disassembly and phagocytic cup closure [42]. Elevated F-actin content was detected in neutrophils from our patient 
(Fig. 4b). Decreased cycling between F-actin and G-actin is expected to result in impaired actin polarization, which was observed in patient T cells and neutrophils (Fig. 5a, b), along with impaired RAC2 polarization in neutrophils (Fig. 5c). The apparent macropinosomes detected in neutrophils from our patient were consistent with the abnormal macropinocytosis reported in patients with RAC2 p.E62K mutations [20]. These abnormalities are established as associated with numerous functions, including cellular migration, intracellular trafficking, and phagocytosis, among others. These results demonstrate that tight regulation of RAC2 activity is pivotal for the control of actin dynamics and actin cytoskeleton reorganization required for physiological cell polarization and macropinocytosis.

\section{Increased Apoptosis in T/B Cells and Neutrophils}

The RAC2 p.G12R mutation is proven to abrogate the survival and differentiation capacities of hematopoietic stem and progenitor cells, because of consequent defects in cellular and mitochondrial networks [23]. Analogously, transgenic mice expressing the constitutively active RAC2 p.G12V or p.Q61L mutations exhibit increased thymocyte and T lymphocyte apoptosis [43]. Further, the RAC2 p.P34H mutation results in increased apoptosis of unstimulated and TCR-stimulated T cells in vitro and EBV-transformed B cells after serum starvation [19]. Mechanistically, these elegant studies have established preliminary and causative roles for RAC2 in lymphopenia. Similarly, significantly increased apoptosis/necrosis of unstimulated, TCR-stimulated, or FASL-induced T cells, and unstimulated or CPG/F(ab) ${ }_{2}$-stimulated B cells, respectively, were observed in vitro in samples from our patient. In addition, we detected increased apoptosis/necrosis of unstimulated and FASL-induced neutrophils, which could not be rescued by LPS stimulation (Fig. 6a). Furthermore, intracellular active caspase 3 expression was markedly increased in leukocytes from the patient (Fig. 6b). Collectively, our data suggest that RAC2 p.P29R leads to increased apoptosis, partly through influencing caspase 3 , which may help to dissect the signaling mechanisms underlying her observed severe peripheral lymphopenia and neutropenia.

\section{Strengthened Expression of RAC2 in Neutrophils and Lymphocytes}

The RAC2 mutants, p.G12R, p.P34H, p.Q61R, p.E62K, and p.N92T, along with the RAC1 p.P29S and p.N92I alterations, have been shown to exist preferentially in a GTP-bound state, as a result of rapid transition from the GDP-bound state, ultimately resulting in hyperactivity and combined immunodeficiency $[19,20,22,23,37-39]$. By contrast, the p.D57N mutation acts in a dominant negative fashion to interfere with RAC1 and RAC2 function, and displayed 10\% GTP-binding ability, relative to the wild-type protein [12-14]. Intriguingly, expression of the homozygous null p.W56X RAC2 mutation was completely absent in cells transfected with the mutant [17]. As no changes in hydrogen bonds or interactions with GDP were predicted to result from the novel mutation reported here, we investigated its influence on RAC2 expression in our patient, and detected increased mRNA and protein levels in neutrophils and lymphocytes (Fig. 7a-d). Hence, we identified altered expression of RAC2 protein arising from this gain-of-function mutation.

\section{Discussion}

In this study, we identified a novel heterozygous RAC2 mutation, c.86C>G (p.P29R), in a Chinese patient diagnosed with combined immunodeficiency. The patient suffered from recurrent respiratory infections and bronchiectasis. Further, she exhibited enhanced ROS production, elevated F-actin content, and large vacuoles in neutrophils, consistent with previous findings associated with dominant activating RAC2 mutations. The mutated P29 residue is within the highly-conserved Switch I domain, and somatic activating mutations located in this codon of the RAC1 and RAC2 genes have been detected in multiple types of human cancer. Moreover, following the ACMG criteria, this p.P29R mutation can be classified as a pathogenic variant. Hence, we identified a de novo heterozygous RAC2 p.P29R deleterious pathogenic mutation. In parallel, we detected increased cytokine production, skewed T lymphocyte phenotype, accelerated apoptosis with augmented intracellular active caspase 3, and impaired polarization of actin in lymphocytes and neutrophils, as well as impaired 
polarization of RAC2 in neutrophils. Overall, our findings broaden the spectrum of known RAC2 gain-of-function mutations and their underlying pathological mechanisms.

The majority of progress in exploring RAC2 function and regulation has been achieved using model mice. A series of murine studies have demonstrated that RAC2 can control apoptosis and cell growth, as well as survival, of hematopoietic cells [44]. Similarly, the RAC2 p.G12R mutation has proven importance for the survival and differentiation capacity of hematopoietic stem and progenitor cells [23]. As mentioned above, RAC2 p.P34H and the p.P29R mutation identified here severely compromise $\mathrm{T}$ and $\mathrm{B}$ lymphocyte and neutrophil survival, by increasing apoptosis in response to various stimuli. Notably, severe T-cell and B-cell lymphopenia are associated with p.D57N and p.W56X mutations in patients, respectively, which is distinguished from increased peripheral T and B cells in RAC2-/- mice $[4,45,46]$. Together, these findings emphasize the importance of tight regulation of RAC2 in humans; however, the mechanisms underlying RAC2 function in human hematopoietic cells have yet to be clearly elucidated.

Various types of RAC2 mutation, including dominant negative, autosomal recessive loss-of-function, and dominant activating gain-of-function, can result in abnormal cytoskeleton reorganization. Efficient cell motility requires both dynamic and regular F-actin polarization, with pseudopodia extending at the front of the cell and retraction at the rear. This physiological status is maintained by restricting active RAC2 to the front of the cell, a function performed by Wiskott-Aldrich syndrome protein (WASP) through its CRIB motif, which removes active RAC2 from the membrane via endocytosis [47]. Further, recent studies have shown that actin depolymerization is crucial for large particle absorption, a process driven by phosphoinositide 3-kinase (PI3K) and the rapid deactivation of RAC and Cdc42, which is essential for the completion of large particle internalization [48]. An elegant study suggested that RAC activation is sufficient to induce membrane ruffling and macropinocytic cup formation, while subsequent RAC deactivation is required for macropinosome closure and further maturation [49]. These findings are consistent with the association of activating RAC2 mutations with increased macropinocytosis and reduced chemotaxis and polarization. We hypothesize that neutrophilia and decreased ROS production, in apparent contrast with neutropenia and increased ROS production alongside macropinocytosis, could be used to facilitate simple preliminary diagnosis of the nature of RAC2 mutations.

RAC2 can exhibit distinctive activities in mouse and human T cells. RAC2 acts on the IFNG promoter to increase IFN- $\gamma$ production and Th1 differentiation [50], consistent with the findings in our patient of increased T-cell cytokine production and skewing of cTfh and non-cTfh subsets toward the Th1-like phenotype. The aberrant differentiation and function of human T cells may contribute to the observed impaired humoral immune responses and inflammatory tissue lesions. Further, the conserved Switch $₫$ domain in RAC guanosine triphosphatases binds to the PI3K catalytic subunit, and there is crosstalk between the RAC and PI3K signaling pathways [51]; therefore, it is intuitive that some features and pathophysiological mechanisms will be shared between them, as dysregulated cytokine production and accumulation by senescent CD8 T lymphocytes have been proven in patients with activated PI3Kס syndrome, in addition to defective NK cell maturation and degranulation, which can be modified in vitro by selective inhibition of p110delta in patients with RAC2 p.P34H mutation $[19,52,53]$. Notably, an inverted CD4/CD8 ratio, decreased TRECs/KRECs, and decreased naïve CD4 and CD8 $\mathrm{T}$ cells can occur in patients with dominant negative, autosomal recessive loss-of-function, and dominant activating mutations of RAC2, highlighting the need for further clarification of the unique roles of this molecule in the human hematopoietic system.

\section{Conclusion}

In conclusion, we report a Chinese patient with a novel heterozygous activating mutation in RAC2, who presented with recurrent pneumonia, bronchiectasis, hypogammaglobulinemia, and severe lymphopenia. Comprehensive immunological assessment indicated that this novel mutation may affect the proliferation, apoptosis, and polarization of leukocytes. The pathogenesis of immunodeficiency in patients with RAC2 mutations remains poorly characterized, and further detailed research is warranted. 


\section{Declarations}

\section{Acknowledgements}

We are grateful to the patient and her family for their continuous cooperation in this study. We thank the members of the laboratory for their technical assistance, and the doctors and nurses for their generous support of this project.

\section{Funding}

This work was supported by the Postgraduate Tutor Team Project of Chongqing Municipal Education Commission (Education Research and Development Program of Chongqing 2019-9).

\section{Competing Interests}

The authors declare no conflict of interest.

\section{Author Contributions}

L.Z., G.L., Z.C., and ZX.D. designed the study and wrote the manuscript; L.Z., Z.C., G.L., WY.L., Q.L., YP.W., XM.C., QY.Y., and ZR.T. performed the experiments; L.Z., Z.C., and G.L. analyzed the data; and G.L. followed the patients; ZY.Z., YF.A., HW.M., and XM.T. offered some crucial clinical data; all the authors reviewed the manuscript before it was submitted.

\section{Data Availability}

The data analyzed in the current study is available from the corresponding author on reasonable request.

\section{Ethics approval}

The study was approved by the Medical Ethics Committee of the Children's Hospital of Chongqing Medical University (Approval number: 030/2013).

\section{Consent to participate}

Informed consent was obtained from the patient and her parents, in accordance with the Declaration of Helsinki.

\section{Consent to publish}

Written informed consent was obtained from all participants included in the study.

\section{References}

1. Heasman SJ, Ridley AJ. Mammalian Rho GTPases: new insights into their functions from in vivo studies. Nat Rev Mol Cell Biol. 2008;9(9):690-701.

2. Mulloy JC, Cancelas JA, Filippi MD, et al. Rho GTPases in hematopoiesis and hemopathies. Blood. 2010;115(5):93647. 
3. Roberts AW, Kim C, Zhen L, et al. Deficiency of the hematopoietic cell-specific Rho family GTPase Rac2 is characterized by abnormalities in neutrophil function and host defense. Immunity. 1999;10(2):183-96.

4. Croker BA, Tarlinton DM, Cluse LA, et al. The Rac2 guanosine triphosphatase regulates $B$ lymphocyte antigen receptor responses and chemotaxis and is required for establishment of B-1a and marginal zone B lymphocytes. J Immunol. 2002;168(7):3376- 86.

5. Jansen M, Yang FC, Cancelas JA, et al. Rac2-deficient hematopoietic stem cells show defective interaction with the hematopoietic microenvironment and long-term engraftment failure. Stem Cells. 2005;23:335-346.

6. Arana E, Vehlow A, Harwood NE, et al. Activation of the Small GTPase Rac2 via the B Cell Receptor Regulates B Cell Adhesion and Immunological-Synapse Formation. Immunity. 2008;28(1):88-99.

7. Savina A, Peres A, Cebrian I, et al. The small GTPase Rac2 controls phagosomal alkalinization and antigen crosspresentation selectively in CD8+ dendritic cells. Immunity. 2009;30(4):544-55.

8. Troeger A, Williams DA. Hematopoietic-specific Rho GTPases Rac2 and RhoH and human blood disorders. Exp Cell Res. 2013;319(15):2375-83.

9. Janssen E, Geha RS. Primary immunodeficiencies caused by mutations in actin regulatory proteins. Immunol Rev. 2019;287(1):121-134.

10. Lougaris V, Baronio M, Gazzurelli L, et al. RAC2 and primary human immune deficiencies. J Leukoc Biol. 2020;108(2):687-696.

11. El Masri R, Delon J. RHO GTPases: from new partners to complex immune syndromes. Nat Rev Immunol. 2021;21(8):499-513.

12. Ambruso DR, Knall C, Abell AN, et al. Human neutrophil immunodeficiency syndrome is associated with an inhibitory Rac2 mutation. Proc Natl Acad Sci U S A. 2000;97(9):4654-9.

13. Williams DA, Tao W, Yang F, et al. Dominant negative mutation of the hematopoietic specific Rho GTPase, Rac2, is associated with a human phagocyte immunodeficiency. Blood. 2000;96(5):1646-1654.

14. Gu Y, Jia B, Yang FC, et al. Biochemical and biological characterization of a human Rac2 GTPase mutant associated with phagocytic immunodeficiency. J Biol Chem. 2001;276(19):15929-38.

15. Routes JM, Grossman WJ, Verbsky J, et al. Statewide newborn screening for severe T-cell lymphopenia. JAMA. 2009;302(22):2465-70.

16. Accetta D, Syverson G, Bonacci B, et al. Human phagocyte defect caused by a Rac2 mutation detected by means of neonatal screening for T-cell lymphopenia. J Allergy Clin Immunol. 2011;127:535-538.

17. Alkhairy OK, Rezaei N, Graham RR, et al. RAC2 loss-of-function mutation in 2 siblings with characteristics of common variable immunodeficiency. J Allergy Clin Immunol. 2015;135(5):1380-4.e1-5.

18. Hodge RG, Ridley AJ. Regulating Rho GTPases and their regulators. Nat Rev Mol Cell Biol. 2016; 17(8):496-510.

19. Lougaris V, Chou J, Beano A, et al. A monoallelic activating mutation in RAC2 resulting in a combined immunodeficiency. J Allergy Clin Immunol. 2019;143(4):1649-1653.e3.

20. Hsu AP, Donkó A, Arrington ME, et al. Dominant activating RAC2 mutation with lymphopenia, immunodeficiency, and cytoskeletal defects. Blood. 2019;133(18): 1977-1988.

21. Sharapova SO, Haapaniemi E, Sakovich IS, et al. Heterozygous activating mutation in RAC2 causes infantile-onset combined immunodeficiency with susceptibility to viral infections. Clin Immunol. 2019; 205:1-5.

22. Smits BM, Lelieveld PHC, Ververs FA, et al. A dominant activating RAC2 variant associated with immunodeficiency and pulmonary disease. Clin Immunol. 2020;212:108248.

23. Lagresle-Peyrou C, Olichon A, Sadek H, et al. A gain-of-function RAC2 mutation is associated with bone-marrow hypoplasia and an autosomal dominant form of severe combined immunodeficiency. Haematologica.

2021;106(2):404-411.

Page $10 / 17$ 
24. Stern H, Donkó A, Shapiro T, et al. A Novel RAC2 Variant Presenting as Severe Combined Immunodeficiency. J Clin Immunol. 2021;41(2):473-476.

25. Yang L, Xue X, Zeng T, et al. Novel biallelic TRNT1 mutations lead to atypical SIFD and multiple immune defects. Genes Dis. 2020;7(1):128-137.

26. Jenks SA, Cashman KS, Zumaquero E, et al. Distinct Effector B Cells Induced by Unregulated Toll-like Receptor 7 Contribute to Pathogenic Responses in Systemic Lupus Erythematosus. Immunity. 2018;49(4):725-739.e6.

27. Boyum A, Lovhaug D, Tresland L, Nordlie EM. Separation of leucocytes: improved cell purity by fine adjustments of gradient medium density and osmolality. Scand J Immunol. 1991;34(6):697-712.

28. Wekell P, Björnsdottir H, Björkman L, et al. Neutrophils from patients with SAPHO syndrome show no signs of aberrant NADPH oxidasedependent production of intracellular reactive oxygen species. Rheumatology (Oxford). 2016;55(8):1489-98.

29. Sottini A, Serana F, Bertoli D, et al. Simultaneous quantification of T-cell receptor excision circles (TRECs) and K-deleting recombination excision circles (KRECs) by real-time PCR. J Vis Exp. 2014;(94):52184.

30. Bylund J, Björnsdottir H, Sundqvist M, et al. Measurement of respiratory burst products, released or retained, during activation of professional phagocytes. Methods Mol Biol. 2014; 1124:321-38.

31. Paccani SR, Boncristiano M, Patrussi L, et al. Defective Vav expression and impaired F-actin reorganization in a subset of patients with common variable immunodeficiency characterized by T-cell defects. Blood. 2005;106(2):626-34.

32. Honda $\mathrm{F}, \mathrm{Kano} \mathrm{H}$, Kanegane $\mathrm{H}$, et al. The kinase Btk negatively regulates the production of reactive oxygen species and stimulation-induced apoptosis in human neutrophils. Nat Immunol. 2012;13(4):369-78.

33. Gabl M, Holdfeldt A, Sundqvist M, et al. FPR2 signaling without $\beta$-arrestin recruitment alters the functional repertoire of neutrophils. Biochem Pharmacol. 2017;145: 114-122.

34. Mao H, Yang W, Latour S, et al. RASGRP1 mutation in autoimmune lymphoproliferative syndrome-like disease. J Allergy Clin Immunol. 2018; 142(2):595-604.e16.

35. Escobar D, Pons J, Clemente A, et al. Defective B cell response to TLR9 ligand (CpG-ODN), Streptococcus pneumoniae and Haemophilus influenzae extracts in common variable immunodeficiency patients. Cell Immunol. 2010;262(2):10511.

36. Bunney TD, Opaleye O, Roe SM, et al. Structural Insights into Formation of an active signaling complex between Rac and phospholipase C Gamma 2. Mol Cell. 2009;34(2):223-33.

37. Hodis E, Watson IR, Kryukov GV, et al. A Landscape of Driver Mutations in Melanoma. Cell. 2012;150(2):251-63.

38. Krauthammer $\mathrm{M}$, Kong $\mathrm{Y}, \mathrm{Ha} \mathrm{BH}$, et al. Exome sequencing identifies recurrent somatic RAC1 mutations in melanoma. Nat Genet. 2012;44(9):1006-14.

39. Kawazu M, Ueno T, Kontani K, et al. Transforming mutations of RAC guanosine triphosphatases in human cancers. Proc Natl Acad Sci U S A. 2013;110(8):3029-34.

40. Richards S, Aziz N, Bale S, et al. ACMG Laboratory Quality Assurance Committee. Standards and guidelines for the interpretation of sequence variants: a joint consensus recommendation of the American College of Medical Genetics and Genomics and the Association for Molecular Pathology. Genet Med. 2015;17(5):405-24.

41. Filippi MD, Harris CE, Meller J, et al. Localization of Rac2 via the $C$ terminus and aspartic acid 150 specifies superoxide generation, actin polarity and chemotaxis in neutrophils. Nat Immunol. 2004;5(7):744-51.

42. Lawson CD, Burridge K. The on-off relationship of Rho and Rac during integrin-mediated adhesion and cell migration. Small GTPases. 2014;5:e27958.

43. Lorès P, Morin L, Luna R, Gacon G. Enhanced apoptosis in the thymus of transgenic mice expressing constitutively activated forms of human Rac2GTPase. Oncogene. 1997;15(5):601-5.

44. Cancelas JA, Williams DA. Rho GTPases in hematopoietic stem cell functions. Curr Opin Hematol. 2009;16(4):249-54. 
45. Guo F, Cancelas JA, Hildeman D, et al. Rac GTPase isoforms Rac1 and Rac2 play a redundant and crucial role in T-cell development. Blood. 2008;112(5):1767-75.

46. Dumont C, Corsoni-Tadrzak A, Ruf S, et al. Rac GTPases play critical roles in early T-cell development. Blood. 2009;113(17):3990-8.

47. Amato C, Thomason PA, Davidson AJ, et al. WASP Restricts Active Rac to Maintain Cells' Front Rear Polarization. Curr Biol. 2019;29(24):4169-4182.e4.

48. Schlam D, Bagshaw RD, Freeman SA, et al. Phosphoinositide 3-kinase enables phagocytosis of large particles by terminating actin assembly through Rac/Cdc42 GTPase activating. Nat Commun. 2015;6:8623.

49. Fujii M, Kawai K, Egami Y, Araki N. Dissecting the roles of Rac1 activation and deactivation in macropinocytosis using microscopic photo-manipulation. Sci Rep. 2013;3:2385.

50. Li B, Yu H, Zheng W, et al. Role of the guanosinetriphosphatase Rac2 in T helper 1 cell differentiation. Science. 2000;288(5474):2219-22.

51. Campa CC, Ciraolo E, Ghigo A, et al. Crossroads of PI3K and Rac pathways. Small GTPases. 2015;6(2):71-80.

52. Tabellini G, Baronio M, Patrizi O, et al. The RAC2-PI3K axis regulates human NK cell maturation and function. Clin Immunol. 2019;208:108257.

53. Nunes-Santos CJ, Uzel G, Rosenzweig SD. PI3K pathway defects leading to immunodeficiency and immune dysregulation. J Allergy Clin Immunol. 2019;143(5):1676-1687.

\section{Tables}

\section{Table 1}

Patient immunoglobulin levels

\begin{tabular}{|lcccc|}
\hline Serum immunoglobulin & $\mathbf{1 1}$ years old & $\mathbf{1 2}$ years $\mathbf{4}$ months old & $\mathbf{1 2}$ years $\mathbf{6}$ months old & Reference range \\
\hline $\operatorname{lgG}(\mathrm{g} / \mathrm{L})$ & $4.45 \downarrow$ & $6.77 \downarrow$ & 9.87 & $8.27-14.17$ \\
\hline $\lg \mathrm{(g} / \mathrm{L})$ & $0.42 \downarrow$ & $0.49 \downarrow$ & $0.68 \downarrow$ & $0.86-1.92$ \\
\hline $\lg M(\mathrm{~g} / \mathrm{L})$ & $1.04 \downarrow$ & $0.91 \downarrow$ & $0.80 \downarrow$ & $1.22-2.56$ \\
\hline $\lg \mathrm{E}(\mathrm{IU} / \mathrm{mL})$ & 1.30 & 3.60 & 2.60 & $0-200$ \\
\hline
\end{tabular}

Table 2

Patient immunological profile 


\begin{tabular}{|c|c|c|c|c|c|c|c|c|}
\hline & \multicolumn{4}{|l|}{11 years old } & \multicolumn{4}{|c|}{12 years 4 months old } \\
\hline & Percentage & $\begin{array}{l}\text { Reference } \\
\text { range }\end{array}$ & $\begin{array}{l}\text { Number/ } \\
\mu \mathrm{L}\end{array}$ & $\begin{array}{l}\text { Reference } \\
\text { range }\end{array}$ & Percentage & $\begin{array}{l}\text { Reference } \\
\text { range }\end{array}$ & $\begin{array}{l}\text { Number/ } \\
\mu \mathrm{L}\end{array}$ & $\begin{array}{l}\text { Reference } \\
\text { range }\end{array}$ \\
\hline T cells & $80.7 \uparrow$ & $\begin{array}{l}57.10- \\
73.43\end{array}$ & $331.0 \downarrow$ & $\begin{array}{l}1325- \\
2276\end{array}$ & 75.3 & $\begin{array}{l}62.06- \\
76.54\end{array}$ & $271.2 \downarrow$ & $\begin{array}{l}1297.20- \\
2479.91\end{array}$ \\
\hline $\mathrm{CD}^{+} \mathrm{T}$ cells & $60.7 \uparrow$ & $\begin{array}{l}21.01- \\
33.94\end{array}$ & $248.9 \downarrow$ & $\begin{array}{l}480- \\
1112\end{array}$ & $59.6 \uparrow$ & $\begin{array}{l}22.50- \\
32.37\end{array}$ & $214.4 \downarrow$ & $\begin{array}{l}508.71- \\
1050.13\end{array}$ \\
\hline $\mathrm{CD}^{+}$naïve & $7.5 \downarrow$ & $\begin{array}{l}41.41- \\
73.04\end{array}$ & $18.7 \downarrow$ & $245-657$ & $4.1 \downarrow$ & $\begin{array}{l}36.05- \\
72.25\end{array}$ & $8.7 \downarrow$ & $\begin{array}{l}232.00- \\
665.14\end{array}$ \\
\hline $\begin{array}{l}\text { CD }^{+} \\
\text {TEMRA }\end{array}$ & $47.6 \uparrow$ & $\begin{array}{l}2.01- \\
21.65\end{array}$ & 118.5 & $12-164$ & $44.2 \uparrow$ & $\begin{array}{l}1.35- \\
21.50\end{array}$ & 94.8 & $\begin{array}{l}10.63- \\
175.36\end{array}$ \\
\hline $\mathrm{CD}^{+} \mathrm{CM}$ & 21.2 & $\begin{array}{l}13.21- \\
37.89\end{array}$ & $52.8 \downarrow$ & $92-287$ & 15.9 & $\begin{array}{l}13.05- \\
39.45\end{array}$ & $34.1 \downarrow$ & $\begin{array}{l}99.72- \\
300.57\end{array}$ \\
\hline $\mathrm{CD}^{+} \mathrm{EM}$ & $23.7 \uparrow$ & $\begin{array}{l}1.53- \\
15.39\end{array}$ & 59.0 & $9-130$ & $35.8 \uparrow$ & $\begin{array}{l}2.00- \\
16.75\end{array}$ & 76.7 & $\begin{array}{l}14.28- \\
156.76\end{array}$ \\
\hline $\mathrm{CD}^{+} \mathrm{T}$ cells & $11.8 \downarrow$ & $\begin{array}{l}24.00- \\
38.72\end{array}$ & $48.5 \downarrow$ & $\begin{array}{l}531- \\
1110\end{array}$ & $12.3 \downarrow$ & $\begin{array}{l}28.47- \\
41.39\end{array}$ & $44.4 \downarrow$ & $\begin{array}{l}621.39- \\
1258.00\end{array}$ \\
\hline CD4 ${ }^{+}$naïve & $6.4 \downarrow$ & $\begin{array}{l}39.72- \\
69.59\end{array}$ & $3.1 \downarrow$ & $294-683$ & $2.3 \downarrow$ & $\begin{array}{l}39.85- \\
71.80\end{array}$ & $1.0 \downarrow$ & $\begin{array}{l}298.99- \\
857.03\end{array}$ \\
\hline $\begin{array}{l}\text { CD }^{+} \\
\text {TEMRA }\end{array}$ & 2.1 & $\begin{array}{l}0.10- \\
1.29\end{array}$ & 1.0 & $0-9$ & 0.6 & $\begin{array}{l}0.07- \\
1.65\end{array}$ & 0.3 & $\begin{array}{l}0.60- \\
14.51\end{array}$ \\
\hline $\mathrm{CD}^{+} \mathrm{CM}$ & 42.7 & $\begin{array}{l}24.24- \\
52.73\end{array}$ & $20.7 \downarrow$ & $165-475$ & 48.0 & $\begin{array}{l}23.25- \\
51.30\end{array}$ & $21.3 \downarrow$ & $\begin{array}{l}218.53- \\
463.39\end{array}$ \\
\hline $\mathrm{CD} 4^{+} \mathrm{EM}$ & $48.9 \uparrow$ & $\begin{array}{l}3.40- \\
11.17\end{array}$ & 23.7 & $24-87$ & $49.1 \uparrow$ & $\begin{array}{l}2.65- \\
9.90\end{array}$ & $21.8 \downarrow$ & $\begin{array}{l}24.20- \\
94.14\end{array}$ \\
\hline $\mathrm{TCRa}^{+} \mathrm{DNT}$ & 2.6 & $\begin{array}{l}0.82- \\
2.91\end{array}$ & 10.6 & $13-48$ & 1.1 & $\begin{array}{l}0.68- \\
2.16\end{array}$ & 2.9 & $\begin{array}{l}11.79- \\
41.44\end{array}$ \\
\hline yठ T & 22.8 & $\begin{array}{l}8.10- \\
20.76\end{array}$ & $93.3 \downarrow$ & $124-388$ & 13.4 & $\begin{array}{l}7.80- \\
23.35\end{array}$ & $36.3 \downarrow$ & $\begin{array}{l}121.10- \\
462.29\end{array}$ \\
\hline B cells & $5.8 \downarrow$ & $\begin{array}{l}9.19- \\
19.48\end{array}$ & $23.9 \downarrow$ & $216-536$ & $7.9 \downarrow$ & $\begin{array}{l}9.23- \\
18.15\end{array}$ & $28.4 \downarrow$ & $\begin{array}{l}247.05- \\
578.16\end{array}$ \\
\hline Memory B & $44.4 \uparrow$ & $\begin{array}{l}8.96- \\
24.09\end{array}$ & $10.6 \downarrow$ & $28-89$ & 16.4 & $\begin{array}{l}8.85- \\
22.90\end{array}$ & $4.7 \downarrow$ & $\begin{array}{l}29.53- \\
89.71\end{array}$ \\
\hline Naïve B & $31.4 \downarrow$ & $\begin{array}{l}51.84- \\
77.61\end{array}$ & $7.5 \downarrow$ & $123-362$ & 62.2 & $\begin{array}{l}44.95- \\
75.80\end{array}$ & 17.7\ & $\begin{array}{l}140.30- \\
380.88\end{array}$ \\
\hline $\begin{array}{l}\text { Transitional } \\
\text { B }\end{array}$ & $11.7 \uparrow$ & $\begin{array}{l}2.50- \\
9.07\end{array}$ & 2.8 & $7-37$ & $13.6 \uparrow$ & $\begin{array}{l}1.75- \\
10.30\end{array}$ & $3.9 \downarrow$ & $\begin{array}{l}5.12- \\
36.61\end{array}$ \\
\hline $\begin{array}{l}\text { Plasmablast } \\
\text { B }\end{array}$ & $12.1 \uparrow$ & $\begin{array}{l}0.70- \\
5.67\end{array}$ & 2.9 & $3-21.0$ & 3.2 & $\begin{array}{l}0.70- \\
7.95\end{array}$ & $0.9 \downarrow$ & $\begin{array}{l}2.48- \\
35.86\end{array}$ \\
\hline NK cells & 12.8 & $\begin{array}{l}10.01- \\
26.98\end{array}$ & $52.5 \downarrow$ & $246-792$ & 15.2 & $\begin{array}{l}7.75- \\
23.47\end{array}$ & $54.76 \downarrow$ & $\begin{array}{l}202.54- \\
583.53\end{array}$ \\
\hline CD4:CD8 & $0.19 \downarrow$ & $\begin{array}{l}0.81- \\
1.66\end{array}$ & & & $0.21 \downarrow$ & $\begin{array}{l}0.92- \\
1.73\end{array}$ & & \\
\hline TREC & & & $3.5 \downarrow$ & & & & $8.3 \downarrow$ & \\
\hline KREC & & & & & & & $24 \downarrow$ & \\
\hline
\end{tabular}


TEMRA, terminally differentiated effector memory helper $\mathrm{T}$ lymphocytes; $\mathrm{CM}$, central memory; EM, effector memory. Naïve, CD45RA ${ }^{+} \mathrm{CD}_{27}^{+}$; TEMRA, CD45RA ${ }^{+} \mathrm{CD} 27^{-}$; CM, CD45RA-CD27+; EM, CD45RA-CD27; TCRa ${ }^{+}$DNT,

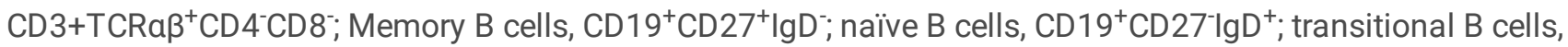
$\mathrm{CD} 19^{+} \mathrm{CD} 24^{++} \mathrm{CD} 38^{++}$; plasmablasts, $\mathrm{CD} 19^{+} \mathrm{CD} 24^{++} \mathrm{CD} 38^{++}$. KRECs, kappa-deleting recombination excision circles; TRECs, T-cell receptor excision circles.

Table 3

Prediction of RAC2 mutation pathogenicity

\begin{tabular}{|lllllll|}
\hline Variant & Provean & Provean & MutationTaster & MutationTaster & PolyPhen-2 & PolyPhen-2 \\
& Score & Prediction & Prediction & Prob & HumDiv & HumVar \\
\hline P29R & -8.054 & Deleterious & Disease causing & 1.000 & 1.0 & 0.986 \\
\hline
\end{tabular}

\section{Figures}

Fig 1

A

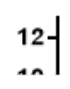

B

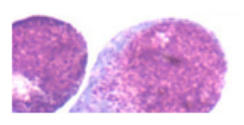

C

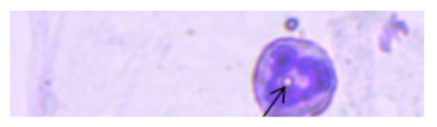

\section{Figure 1}

Patient clinical features. (a) Absolute white blood cell counts over time. Large vacuoles (arrows) visible by light microscopy within neutrophils from bone marrow (b) and peripheral blood (c), and low density neutrophils from isolated peripheral blood mononuclear cells (PBMC) (d). (e) Computed tomography (CT) scans of the patient at 11 years old. Axial (left) and frontal (right) chest CT images showing extensive asymmetrical bronchiectasis, with peripheral inflammation in both lungs 
Fig 2
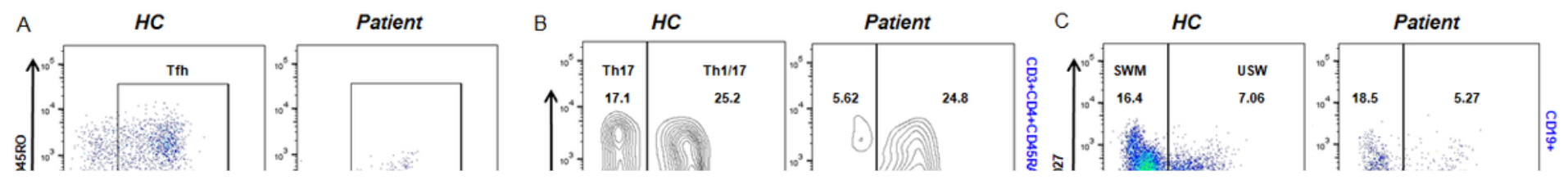

\section{Figure 2}

Skewed peripheral T and B cell subsets in patient samples. The patient showed: (a) an increased percentage of activated Tfh cells; (b) skewed differentiation of Tfh and non-Tfh cells toward the Th1-like phenotype; and (c) increased percentages of double negative (DN) and DN2 cells. CD19+ $B$ cell populations were defined by $\operatorname{lgD}$ and CD27 as follows: $\lg D^{-} \mathrm{CD}^{2} 7^{+}$, switched memory (SWM); $\lg \mathrm{D}^{+} \mathrm{CD} 27^{+}$, unswitched memory (USW); $\lg \mathrm{D}^{+} \mathrm{CD} 27^{-}$, naïve (NAV); and $\operatorname{lgD}{ }^{-} \mathrm{CD}_{27}{ }^{-}$, DN. Most DN B cells in the patient lacked CD21 (DN2 cells). Data are from one representative of two independent experiments

Fig 3

A

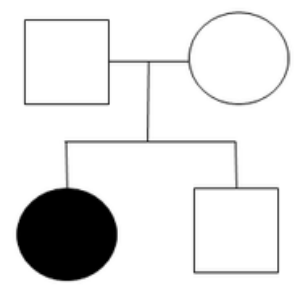

C

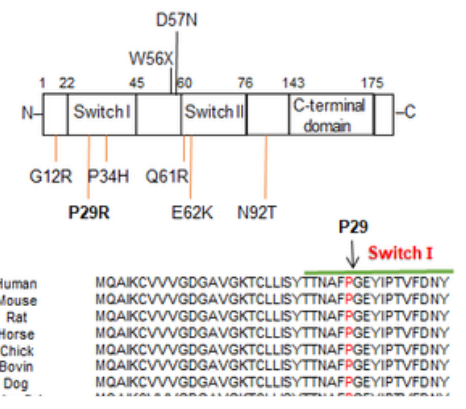

Figure 3 
A novel de novo mutation identified within the highly-conserved RAC2 Switch I domain. (a) Family pedigree. (b) Sanger sequencing of RAC2 exon 2 from whole blood, isolated peripheral blood mononuclear cells, and neutrophil samples showing the $c .86 \mathrm{C}>\mathrm{G}$ mutation in the patient and wild-type sequence in her parents. (c) Schematic representation of the RAC2 protein and its mutations (upper), as well as amino acid alignment of the highly-conserved Switch I domain across different species (lower). All mutations identified to date are indicated in the protein domains; reported dominant activating monoallelic mutations are depicted as red vertical bars

Fig 4

A

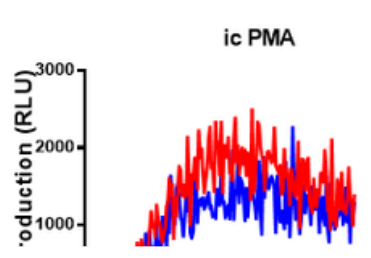

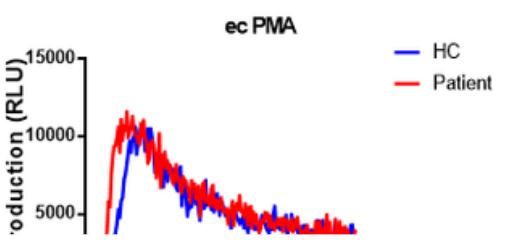

B

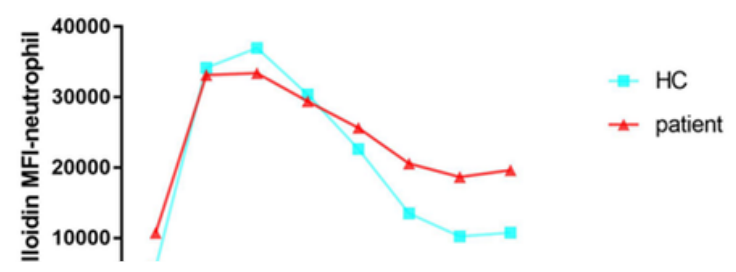

Figure 4

Expanded intracellular NADPH-oxidase-derived reactive oxygen species (ROS) production and elevated F-actin content accumulation in granulocytes. (a) Extracellular ROS production by granulocytes was measured as isoluminol-amplified chemiluminescence, and luminol-amplified chemiluminescence assessed to measure intracellular ROS in response to different stimuli. (b) F-actin content assessed by determining the median fluorescent intensity (MFI) of permeabilized neutrophils stained with phalloidin-FITC. Data are from one representative of two independent experiments

\section{Figure 5}

Impaired actin and RAC2 polarization. (a) Diminished F-actin cap formation in isolated purified CD3 T cells in response to CD3/CD28 co-stimulation, measured by confocal microscopy of cells labeled with phalloidin-FITC. (b) Decreased F-actin reorganization in neutrophils in response to PMA. (c) Reduced RAC2 polarization in neutrophils in response to PMA

\section{Figure 6}

Increased apoptosis/necrosis of T/B cells and neutrophils. (a) Percentages of apoptosis/necrosis of neutrophils (upper left), B cells (upper right), CD4 T cells (bottom left), and CD8 T cells (bottom right) in response to different stimuli, as 
determined by Annexin V and 7-AAD staining and analyzed by flow cytometry. (b) Intracellular expression of active caspase 3 in neutrophils, B cells, and CD4 and CD8 T cells. Data are representative of one of two independent experiments

Fig 7

A

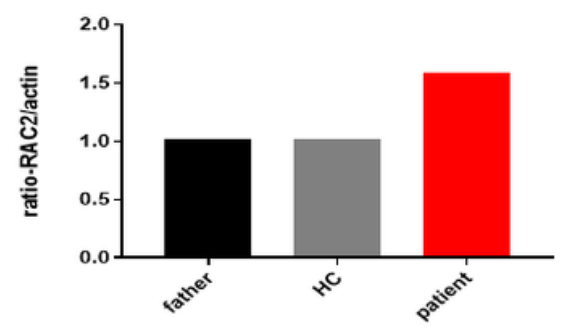

C

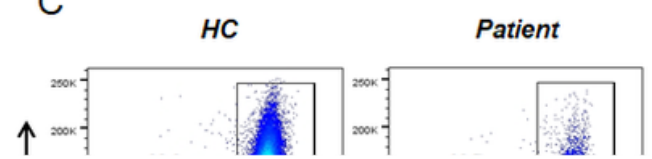

B

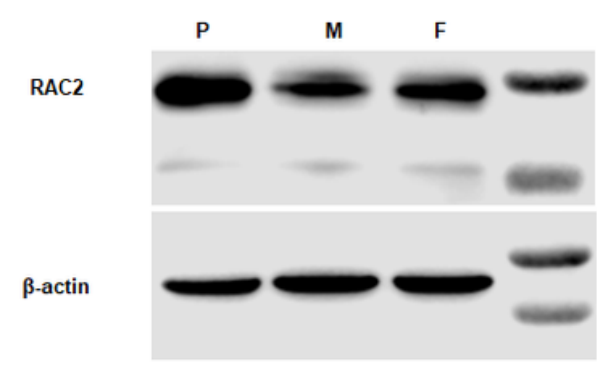

D

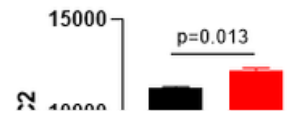

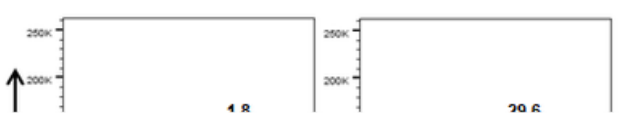

\section{Figure 7}

Increased RAC2 expression in neutrophils. (a) RT-PCR of RAC2 showed increased transcript levels in the patient (vs. actin) (b) Detection of RAC2 expression in isolated neutrophils by western blot analysis. (c) Detection of RAC2 expression in isolated neutrophils by flow cytometry. (d) Detection of RAC2 expression in lymphocytes by flow cytometry. Data are representative of one from two or three independent experiments

\section{Supplementary Files}

This is a list of supplementary files associated with this preprint. Click to download.

- supplementaldata.pdf 